\title{
Estudo comparativo da atividade eletromiográfica da porção longa do bíceps braquial em praticantes de musculação antes e após alongamento passivo Comparative study of electromyographic activity of the long head of the brachial biceps in bodybuilders before and after passive stretching
}

\author{
Danilo Ferreira da Silva*, Diego Felipe Tenório Correia*, Rafael Ferreira Romualdo*, \\ Geraldo Magella Teixeira, Ft., D.Sc.**
}

*Acadêmicos de Educação Física da Faculdade Estácio de Alagoas (SESAU), MaceiólAL, ${ }^{*}$ Orientador, Professor do curso de Educação Física da Universidade Estácio - FAL, MaceiólAL

\section{Resumo}

Objetivo: Avaliar comparativamente o efeito imediato da técnica de alongamento passivo na ativação elétrica da porção longa do bíceps braquial do membro superior dominante em praticantes de musculação há pelo menos 1 ano. Material e métodos: Estudo observacional, do qual participaram 35 indivíduos do sexo masculino, maiores de 18 anos, praticantes de musculação há pelo menos 1 ano. Todos foram avaliados por meio da eletromiografia de superfície antes e após o alongamento passivo do músculo bíceps braquial do membro superior dominante. Resultados: Antes da realização do alongamento passivo, a média da atividade elétrica muscular do bíceps braquial durante a flexão do cotovelo contra resistência de $1 \mathrm{~kg}$ foi de $175,84 \mathrm{mV}$ e após a aplicação do alongamento passivo, para a execução da mesma tarefa, a média foi de $92,38 \mathrm{mV}$. Conclusão: O estudo sugere que a técnica de alongamento passivo pode ser eficaz na diminuição da atividade elétrica muscular em praticantes de musculação durante a execução de exercícios resistidos.

\begin{abstract}
Objective: To evaluate comparatively the immediate effect of passive stretching technique in the electrical stimulation of the long portion of brachial biceps of dominant upper limb in bodybuilders for at least one year. Methods: This observational study was composed of 35 male individuals, over 18 years old, who performed bodybuilding for at least one year. All were evaluated using surface electromyography before and after passive stretching of the brachial biceps muscle of dominant upper limb. Results: Before performing passive stretching, the average of muscle electrical activity of the brachial biceps during elbow flexion against resistance of $1 \mathrm{~kg}$ was $175.84 \mathrm{mV}$ and after passive stretching, to perform the same task, the average was $92.38 \mathrm{mV}$. Conclusion: The study suggests that the passive stretching technique can be effective in reducing muscle electrical activity in bodybuilders during resistance exercises.
\end{abstract}

Key-words: brachial biceps, passive stretching, surface electromyography.

Palavras-chave: bíceps braquial, alongamento passivo, eletromiografia de superfície.

Recebido em 13 de novembro de 2014; aceito em 30 de dezembro de 2014.

Endereço para correspondência: Diego Felipe Tenório Correia, Rua Aurélio Cavalcante, 65 Gruta de Lourdes 57052-488 Maceió AL, E-mail: diegof.tenorioc@gmail.com 


\section{Introdução}

De acordo com Weineck [1], a musculação, historicamente, era atividade privada de uma determinada categoria de homens que tinham necessidade de impressionar por meio da imagem física. Com a cientificização da musculação foi possível constatar que essa atividade pode, além de benefícios estéticos, proporcionar bem-estar, melhoria no desempenho do aparelho locomotor, além de trazer ganhos cognitivos [2]. Ramos [3], ao abordar a questáo, coloca que praticantes de musculação que possuem bom recrutamento de unidades motoras possuem vantagens em relação à independência funcional quando comparados com não praticantes.

De acordo com Weineck [1], o processo de ganho de força pela contração muscular envolve diversas proteínas celulares e sistemas de produção de energia e, de acordo com Ferreira [4], a actina e miosina são as proteínas mais importantes nesse processo e participam da contração muscular quando o cálcio citosólico $([\mathrm{Ca} 2+] \mathrm{i})$ aumenta, disparando uma série de eventos moleculares que levam à interação entre miosina e actina, ocorrendo o deslizamento desta última sobre os filamentos grossos e o encurtamento dos sarcômeros em série. Por outro lado, Ramos [3] constata que o processo de ganho de massa muscular pode favorecer o encurtamento muscular e que este encurtamento pode ser influenciado por fatores endógenos; a saber: gênero e idade e fatores exógenos; tais como: temperatura ambiente e hora do dia.

Em relação ao gênero, Costa [5] afirma que, de modo geral, as mulheres, por interferências hormonais, apresentam maior flexibilidade, uma vez que estas apresentam elevada taxa de estrógeno quando comparadas aos homens. Esse mesmo autor estabelece relação do estrógeno e hidratação tecidual e maleabilidade tecidual. Zakharov [6] coaduna com o autor suprarreferido ao confirmar que a supremacia das mulheres, na mobilidade muscular, comparada a dos homens é de $20 \%$ a $30 \%$, não significando que homens bem treinados não sejam capazes de possuir elevado nível de flexibilidade.

Em relação à idade, Achour \& Junior [7] afirmam que com o decorrer dos anos a flexibilidade vai se deteriorando. Isso ocorre em função da diminuição do fornecimento do fluxo sanguíneo e diminuição da capacidade do músculo em reter água. A flexibilidade é maior quanto mais jovem for o sujeito, assim sendo o treinamento da flexibilidade deve ser proposto desde a infância $[1,8]$ no sentido de manter a flexibilidade máxima quando adulto.

No que diz respeito à temperatura e hora do dia, Contursi [9] afirma que os componentes articulares e musculares possuem melhor desempenho quando expostos a temperaturas mais elevadas e para Weineck [2] durante o período matutino, por causa da baixa temperatura corporal a flexibilidade é menor quando comparado com o período vespertino.

Segundo Bompa [10], quando realizados de maneira adequada, os alongamentos são eficazes no ganho de flexibilidade e trazem benefícios, tais como: redução das tensôes musculares; relaxamento corporal; maior consciência corporal; fluidez nos movimentos; prevenção de lesóes; preparam o corpo para atividades físicas e ativam a circulação.

O alongamento passivo ocorre quando o profissional manipula em excentricidade o músculo do aluno; ainda de acordo com o mesmo autor, trata-se de um alongamento eficiente, pois permite relaxar com eficiência a musculatura, desde que realizado por pelo menos 10 segundos [11]. Achour Junior [7] concorda com J. Alter [11] e afirmam que o alongamento passivo é fundamental para diminuir as possibilidades de lesóes e tem como objetivo a recuperação ou manutençáo da flexibilidade; mas propõe ampliação do tempo em excentricidade e assume ser necessário, para obtenção de alongamento eficaz, manter posição de excentricidade por período que pode variar de 10 segundos até alguns minutos.

A eletromiografia de superfície (EMG-S) é um método biomecânico não invasivo que permite o estudo em tempo real da funçáo elétrica muscular pela análise do sinal elétrico emanado durante a atividade muscular [12]. Essa técnica possibilita analisar o tempo de ativação muscular e se há pouca ou muita atividade contrátil do músculo [13], sendo considerado um instrumento de avaliação muito útil em atividades musculares [14]. 
Diante do exposto pretendeu-se, com a presente proposta, averiguar comparativamente a atividade eletromiografia da porçáo longa do bíceps braquial em praticantes de musculaçáo antes e após o alongamento passivo ao realizarem contração isotônica resistida com carga de $1 \mathrm{~kg}$.

\section{Material e métodos}

Após a aprovação do protocolo de Estudo pelo Comitê de Ética em Pesquisa (CEP), Universidade Estadual de Ciências da Saúde de Alagoas, protocolo no 902/08 foram analisados 35 indivíduos do sexo masculino, praticantes de musculação há pelo menos 1 ano, maiores de 18 anos e que náo tinham histórico de queixas álgicas nos membros superiores.

Após a seleção dos sujeitos foi feita a coleta do sinal eletromiográfico da porçáo longa do músculo bíceps braquial do lado dominante durante a contração isotônica contra resistência com carga de $1 \mathrm{~kg}$ para uma única repetição. Para a aquisição do sinal eletromiográfico foi utilizado aparelho Miotec $^{\oplus}$ de 02 canais e eletrodos de superfície de cloreto de prata, circulares e pré-geldados da marca Meditrace ${ }^{\oplus}$. O modelo utilizado foi o Miotool 200 USB, com 14 bits de resolução, ruído < 2 LSB, taxa de aquisição por canal de 2000 amostras/segundo, rejeição de modo comum de 110 decibéis $(\mathrm{dB})$, isolamento de 3000 volts, impedância de entrada de $1010 \Omega \| 2 \mathrm{pF}$ e filtro Butterworth passa alta 1 polo de $0,1 \mathrm{~Hz}+$ Butterworth passa baixa 2 polos de $500 \mathrm{~Hz}$, com ganho fixo de 100x.

Foi seguido o protocolo recomendado por Seniam [15], após assepsia da região com álcool a $70 \%$ e abrasão da pele com uma lixa $180 \mathrm{~mm}$, o primeiro eletrodo foi posicionado no ponto mediano entre a origem e inserção do músculo em questáo e o segundo eletrodo de referência, no epicôndilo lateral do úmero do lado dominante. Foi tomado como ponto de referências da origem muscular em questáo o tubérculo supraglenoidal e o ponto de referência de inserção a tuberosidade radial [16].

$\mathrm{Na}$ primeira etapa foi solicitado ao participante realizar uma única flexão em toda amplitude de movimento (ADM) do cotovelo do lado dominante a partir da posição anatômica. Essa contração foi realizada de forma isotônica e contra resistência com halter de $1 \mathrm{~kg}$; foi aplicada uma força manual no ombro do participante no sentido de impedir compensaçóes da articulação do ombro. O sinal eletromiográfico obtido passou por um filtro analógico passa-banda de 20-450 Hz [17] e, em seguida, foi transformado analogicamente em valores RMS (Root Mean Square), dos quais foi extraído o valor médio da amplitude do sinal processado, representado por microvolts $(\mu \mathrm{V})$. Convém ressaltar que não houve qualquer interesse em fazer estudo interindivíduos e sim intraindivíduos, de modo que nenhuma normalização estatística foi necessária.

A segunda etapa foi realizada por outro pesquisador devidamente treinado e constou dos seguintes procedimentos: retirada dos eletrodos e aplicação da técnica de alongamento passivo do músculo bíceps braquial. Cada exercício foi realizado com três séries de 30 segundos com intervalo de 30 segundos entre as séries $[7,11]$. Os participantes foram instruídos a relatar imediatamente qualquer desconforto surgido durante a manobra. Os participantes foram instruídos a permanecerem sentados em uma cadeira, com as costas bem apoiadas e joelhos fletidos a 90 graus, o colaborador foi instruído a estender o ombro dominante, com o cotovelo também estendido e pronado. O pesquisador exerceu força no sentido de amplificar a ADM do ombro em extensão e impedir a flexão do cotovelo e compensação com o tronco.

Decorridos 5 minutos, período de intervalo, o participante foi submetido à terceira etapa, que consistiu em uma nova avaliação, pelo examinador 1 , da análise do sinal eletromiográfico do músculo em questão. Obedecendo aos mesmos critérios descritos na primeira etapa.

\section{$\overline{\text { Resultado e discussão }}$}

Conforme a tabela abaixo a amostra foi composta de trinta e cinco indivíduos do sexo masculino com média de idade de 23,29 $\pm 2,43$ anos, peso médio de $85,04 \pm 1,98 \mathrm{~kg}$ e média de altura de $1,79 \pm 7,12 \mathrm{~cm}$ e IMC médio de $17,12 \% \pm 1,71$. 
Tabela I - Média $\pm D P$ das características da população $(n=35)$.

\begin{tabular}{lcc}
\hline \multicolumn{1}{c}{ Características } & Média & DP \\
\hline Idade & 23,29 & 2,43 \\
Peso $(\mathrm{kg})$ & 85,04 & 1,98 \\
Altura $(\mathrm{m})$ & 1,79 & 7.12 \\
IMC & 17,12 & 1,71 \\
\hline Fonte: Autores 2013 & &
\end{tabular}

Esses resultados estão de acordo com os dados encontrados por Konsel et al. [18] quando os autores propuseram estudar o comportamento eletromiográfico da porção longa do bíceps braquial em diferentes posiçóes do ombro .

Em relação à atividade elétrica do musculo bíceps braquial antes da atividade de alongamento passivo, o presente estudo mostrou que a média da atividade muscular foi de $175,85 \mathrm{mV}$ e apoio de $92,38 \mathrm{mV}$ com valor da significância de 0,001 . Tais dados podem ser no gráfico abaixo.

Gráfico 1 - Comparação da amplitude da onda eletromiográfica (recrutamento muscular) em $\mathrm{mV}$ do músculo biceps braquial dominante contra resistência antes e após o alongamento passivo $(\alpha=0,001)$.

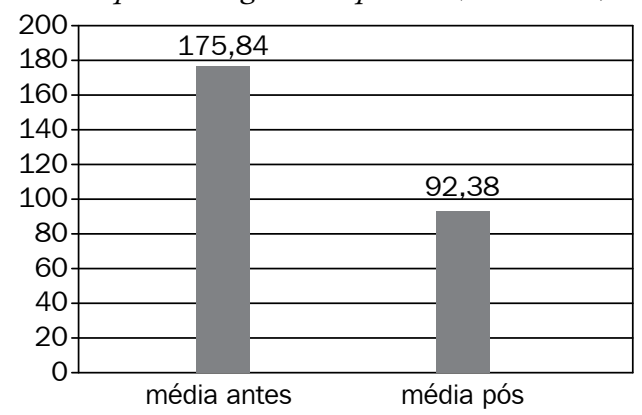

Fonte: Autores 2013

Esses achados estáo em desacordo com os dados encontrados por Gonçalves et al. [19], pois os autores afirmam que a atividade elétrica muscular parece não ser influenciada pelo alongamento. Porém vale salientar que nesse estudo, diferentemente do presente estudo, os autores supracitados usaram a técnica de alongamento ativo. Acredita-se que as diferenças encontradas são decorrentes das diferenças entre as modalidades de alongamento. Já Branco et al. [20] encontram em estudos dados semelhantes ao estudos aqui apresentados. Dados semelhantes podem ser encontrados nos estudo propostos por Cunha et al. [21].

\section{Conclusão}

O estudo sugere que técnica de alongamento passivo pode ser eficaz na diminuição da atividade muscular do bíceps braquial em praticantes de musculação.

\section{Referências}

1. Weineck J. Biologia do esporte. $7^{\mathrm{a}}$ ed. São Paulo: Manole; 2005. 758p.

2. Weineck J. Atividade física e esporte: para quê? São Paulo: Manole; 2003. 254p.

3. Ramos AT. Treinamento de força na atualidade. Rio de Janeiro: Sprint; 2000. 115p.

4. Ferreira AT. Physiology of muscular contraction. Rev Neurociências 2005;13(3)

5. Costa MG. Ginástica localizada. Rio de Janeiro: Sprint; 1996.338p.

6. Zakharov A. Ciência do treinamento desportivo. Traduzido por: Gomes AC. Rio de Janeiro: Grupo Palestra Sport; 1992. 338p.

7. Achour Junior A. Alongamento e flexibilidade: definiçõos e contraposições. Revista Brasileira de atividade Física \& Saúde 2007;12(1):54-8.

8. Tubino G. Metodologia científica do treinamento desportivo. 11a ed. São Paulo: Ibrasa; 1984.435p.

9. Contursi TLB. Flexibilidade e alongamento. Rio de Janeiro: Sprint; 1986. 162p.

10. Bompa TO. Periodização: teoria e metodologia do treinamento. São Paulo: Phorte; 2002.

11. Alter J. Alongamento para os esportes. Rio de Janeiro: Guanabara Koogan; 2001.

12. Amorin LJ. Análise eletromiográfica durante o movimento de pega de objetos para atividades de vida diária. In: Congresso Brasileiro de Biomecânica: Anais do X Congresso Brasileiro de Biomecânica; 2003; Ouro Preto-MG. [S.1.]:[s.n.]; 2003.p.66-70.

13. Souza L. Análise eletromiográfica dos efeitos do alongamento estático passivo no músculo sóleo [Monografia]. Cascavel: Curso de Fisioterapia da Unioeste; 2004.

14. Hou C-R, Tsai L-C, Cheng K-F, Chung K-C, Hong C-Z. Immediate effects of various physical therapeutic modalities on cervical myofascial pain and trigger-point sensitivity. Arch Phys Med Rehabil 2002;83:1406-14.

15. Seniam Group. Recommendations of Sensor Locations [Internet]. Netherlands. [citado 2009 Jan 20]. Disponível em: URL: http://www.seniam.org. 
16. Dangelo JG, Fatinni CA. Anatomia humana sistêmica e segmentar. $3^{a}$ ed. Rio de Janeiro: Atheneu; 2007. 763 p.

17. De Luca CJ. A Practicum on the use of surface EMG signals in movement sciences. Section 2: Technology and the Quality of the EMG Signal. Delsys [Internet]. 2008. [citado 2009 Jan 22]. Disponível em: URL: http://www.delsys.com.

18. Von Konsel M, Castro FS, Cruz D, Fleig T. Análise eletromiográfica da porção longa do bíceps braquial em diferentes posiçóes do ombro. Brazilian Journal of Biomotricity 2009;3(2):167-76.

19. Gonçalves RB, Marques JC, Monte-Raso VV, Zamarioli A, Carvalho LC, Fazan VPS. Efeitos da aplicação do laser de baixa potência na regeneração do nervo isquiático de ratos. Fisioter Pesqui 2010;17(1):34-39.

20. Branco VR, Negrão Filho RF, Padovani CR, Azevedo FM, Alves N, Carvalho AC. Relação entre a tensão aplicada e a sensação de desconforto nos músculos isquitibiais durante o alongamento. Rev Bras Fisioter 2006;10(4):465-72.

21. Cunha APN, Marinho PEM, Silva TNS, França EET, Amorim Filho VCG, Andrade, AD. Efeito do alongamento sobre a atividade dos músculos inspiratórios no DPOC. Saúde Rev 2005;7(17):13-9.

\section{Assine já!}

Revisto brosilioirta de F ISIOLOGIA DO EXERCIÍCI O Brazilian Journal of Exercise Physiology Órgão Oficial da Sociedade Brasileira de Fisiologia do Exercício
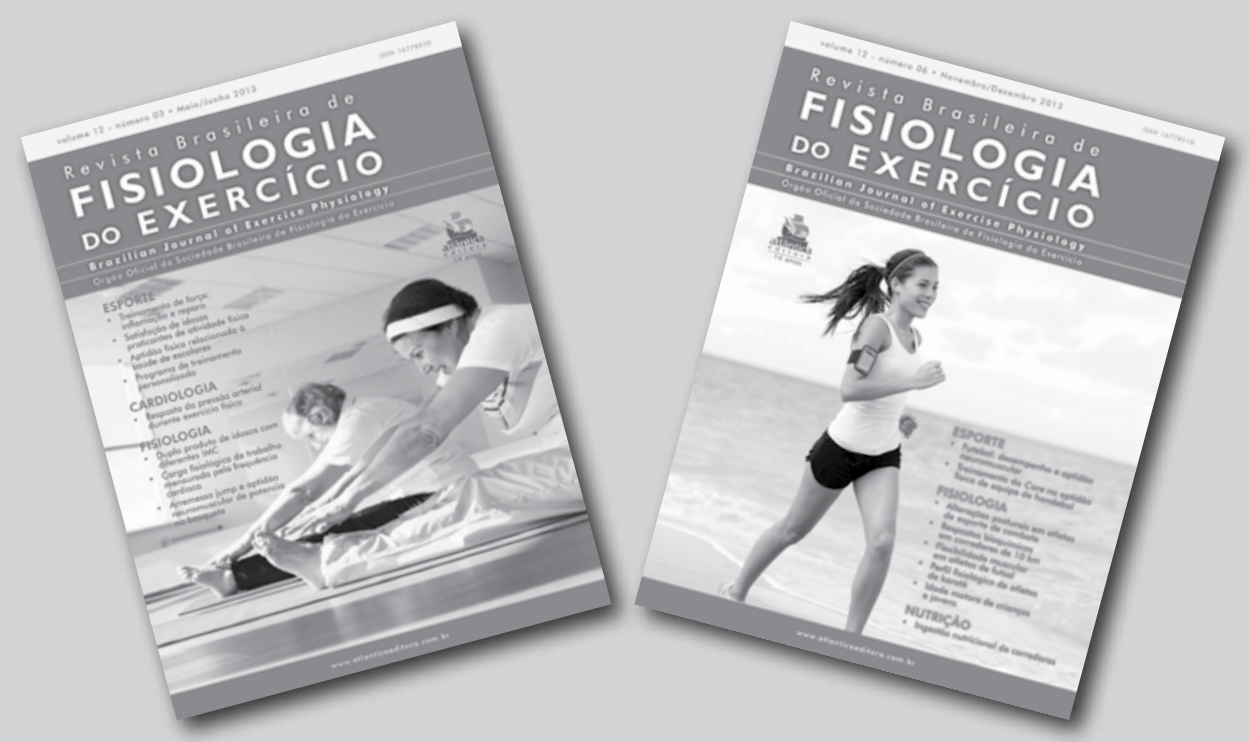

Tel: (11) 3361-5595 / assinaturas@atlanticaeditora.com.br 